\title{
INDICADORES DE SUSTENTABILIDAD DEL MANEJO DE SUELOS EN LA PRODUCCIÓN DE ARVEJA (Pisum sativum L.)
}

\section{INDICATORS OF SUSTAINABILITY OF THE SOIL MANAGEMENT IN THE PEA (Pisum sativum L.) PRODUCTION}

\author{
Agustín Perales ${ }^{1}$, Oscar Loli ${ }^{2}$, Julio Alegre ${ }^{3}$ y Félix Camarena ${ }^{4}$
}

\begin{abstract}
Resumen
Se realizó el diagnostico del manejo de suelos en la producción de arveja (Pisum sativum L.) en el Distrito de Manzanares, Provincia de Concepción, Región y Departamento de Junín, Perú, a través de la investigación participativa, con el objetivo de identificar las zonas homogéneas de producción, caracterizar los sistemas de manejo, determinar los indicadores de sustentabilidad, el índice de sustentabilidad y puntos críticos del manejo de suelos. Se identificaron dos zonas homogéneas de producción, una con riego y otra en secano. En la primera zona prevalecen los sistemas de cultivo con fertilización química y rotación con leguminosas. En la segunda zona prevalecen los sistemas de cultivos con descanso corto (1 año) y con fertilización orgánica. El manejo de suelos en ambas zonas se caracteriza por la remoción de los residuos de los cultivos, reducida aplicación de fertilizantes orgánicos o inorgánicos y deficiente rotación de cultivos, a estos se suman la labranza intensiva y riego inadecuado en la zona conducida bajo riego. Los indicadores de sustentabilidad del manejo de suelos en la producción de arveja seleccionados fueron: El carbono y Nutrientes del suelo, densidad aparente de la capa arable, cobertura vegetal, la presencia de malezas, el rendimiento y nodulación simbiótica de la arveja, la incidencia de plagas y enfermedades. Los sistemas de manejos de suelos en ambas zonas de producción identificadas no fueron sustentables. Los puntos críticos identificados en ambas zonas fueron el manejo de cobertura vegetal, fertilizantes, labranza y rotación de cultivos.
\end{abstract}

Palabras clave: Indicadores de Sustentabilidad, manejo de suelos, arveja, Pisum sativum

\begin{abstract}
A diagnosis of the soil management in pea production, in the district of Manzanares, Concepción - Junín, Perú was carried out, through of participatory research, with the objective of identifying homogeneous zones of production, characterizing systems of management; determining indicators of sustainability, sustainability index and critical points of soil management in pea production. Two homogeneous zones of production were indentified, with and without irrigation. In the first zone, cultivation systems with chemical fertilization and leguminous rotation prevail. In the second one, cultivation systems with short rotation (one year) and organic fertilization systems prevail. Soil management in both areas is characterized by crop residues removal, reduced application of organic or inorganic fertilizers and poor crop rotation. Added to these are intensive cultivation and improper irrigation in the area under irrigation. Identified indicators of soil management sustainability in pea production were: organic carbon and soil nutrients, apparent density of the topsoil, vegetal cover, weeds, yield and pea symbiotic nodulation, and plagues and diseases incidence. Systems of soil management in both zones were not sustainable. The critical points identified in the soil management for both zones were: vegetal cover management, fertilization, tillage, and cultivation rotation.
\end{abstract}

Key words: Sustainability indicators, soil management, Pea, Pisum sativum

\section{Introducción.}

El suelo es un recurso natural muy valioso para garantizar el sustento para la población humana, así como para generar ingresos sostenidos para un país; su uso eficiente y sustentable posibilita una estabilidad política internacional en el largo plazo.

A pesar de la importancia socio económica, el suelo no tiene la atención necesaria, por el contrario tanto la presión demográfica y las diversas actividades que se realizan sobre este recurso, han acelerado su degradación física, química y biológica, con consecuencias sociales y económicas negativas, tales como, la reducción de la productividad, la pérdida de ingresos, el desplazamiento de familias a las ciudades, la pérdida de valores culturales, el aumento de la vulnerabilidad de la población a conflictos sociopolíticos y armados (Nario et al., 2001; Santillana, 2006).

Ante esta situación, en medio de una conciencia ambiental que crece, la practica de la agricultura 
Julio - Diciembre 2009

sustentable aparece como un desafío vital para quienes habiten esta aldea global en el siglo XXI (Cufre et al., 2002). Entonces, si la agricultura va a ser sustentable, esta deberá ser: a) Suficientemente productiva, capaz de alimentar a una población en aumento, consecuentemente mayores rendimientos deben ocurrir y serán el resultado de mejores sistemas de manejo de suelos (SMS); b) Económicamente viable (evaluando todos los costos); c) Ambientalmente o Ecológicamente adecuada (que conserve la base de recursos naturales y preserve la integridad del ambiente a nivel local, regional y global); d) Cultural y socialmente aceptable; y e) Técnicamente apropiado (Nuñez, 2005).

Los Sistemas de Manejo de los Suelos, vienen a ser el uso eficiente del suelo a través de prácticas o técnicas para la producción de una determinada especie vegetal. El uso eficiente y sustentable del recurso suelo permitirá revertir las consecuencias negativas de su deterioro físico químico biológico y posibilitará una estabilidad política internacional en el largo plazo.

La gestión adecuada de los suelos, esta relacionado al manejo de la cobertura y residuos vegetales, a la biodiversidad y rotación de cultivos, a la práctica de la fertilización y a los sistemas de labranza y riego (Nario et al., 2001). Entonces, conocer el sistema de manejo de suelos en la producción de las especies vegetales es muy importante si se quiere que esta sea sustentable. Al respecto aun han sido poco los esfuerzos para evaluar que tan sustentables son los Sistemas de Manejo de los Suelos en la producción agrícola.

Consecuentemente, en el marco de la agricultura sustentable, uno de los desafíos que los científicos se enfrentan es recuperar, mejorar y conservar la calidad del suelo. Por ello una de las primeras acciones es avanzar en el conocimiento de los sistemas de manejo de suelos. La gestión de la calidad del suelo en un agroecosistema, responde a un gran número de prácticas agrícolas, tecnologías que vienen de un conocimiento acumulado por las generaciones e implementadas según la tipología de la agricultura. La caracterización de estas tecnologías en cada agroecosistema, y la determinación de las características más importantes del terreno es una preocupación del usuario para poder usar con más acierto el preciado recurso suelo (Fitz, 1993; USDA, 1994), y representa un elemento importante para entender las lógicas de manejo del suelo que influyen en su calidad $y$ es un medio para analizar el funcionamiento de un determinado sistema de producción (Altiere \& Nicholls, 2007).

Para lograr el propósito señalado, es necesario entender la gestión de la calidad del suelo, evaluar el grado de crisis del sistema, determinar el índice de sustentabilidad, planificar y establecer el manejo sostenible del recurso suelo, a través de estrategias tecnológicas y practicas agrícolas adecuadas, a fin de asegurar una producción de calidad y sustentable (Apollin \& Eberhart, 1999)

Para evaluar la sostenibilidad o grado de crisis de un sistema de manejo de suelos es necesario utilizar indicadores confiables, sensibles y dependientes, que ayuden a detectar o determinar los puntos críticos del sistema, que reflejen los cambios o innovaciones hechas en las tecnologías de manejo de la calidad de los suelos, así como sus efectos dentro del agroecosistema (Díaz et al., 2005)

La aplicación de indicadores, es un medio adecuado para detectar puntos críticos de la sostenibilidad, establecer sus causas y proponer soluciones a mediano plazo. En otras palabras, los indicadores sirven para tomarle el pulso al agroecosistema (Altiere \& Nicholls, 2005)

Por otro lado, la arveja, junto con el fríjol y el haba son las especies de legumbres de mayor importancia en el mundo y en el Perú. La arveja por su distribución extensa en el territorio nacional, con 28 889 ha de superficie sembrada en la campaña 2005 (MINAG, 2007) es considerada como un cultivo que justifica su estudio detallado, y desde un punto de vista social, ella beneficia económicamente a un gran número de familias; nutricionalmente es una fuente importante de carbohidratos (6.3\% en verde) y proteínas (24.1\% en seco) que la convierte en un complemento ideal de la alimentación humana; agrícolamente mejora la fertilidad de los suelos, por su capacidad de fijar el nitrógeno atmosférico hasta 85 kg por hectárea por año (CARITAS, 2004) y porque es un cultivo muy recomendado dentro de un programa de rotación de cultivos. Sin embargo los agricultores tienen serias dificultades para asegurar una buena producción, principalmente debido a sistemas de manejo de suelos (SMS) inadecuados, siendo necesario conocer las limitaciones de carácter edáfico y si los productores están manejando adecuadamente los suelos donde la arveja es cultivada.

En este contexto se realizó el diagnóstico de los sistemas de manejo de suelos en la producción de arveja (Pisum sativum L), con el objetivo de identificar las zonas homogéneas de producción, caracterizar los sistemas de manejo, seleccionar indicadores de sustentabilidad, determinar el índice de sustentabilidad y los puntos críticos del sistema de manejo de los suelos en la producción de arveja.

\section{Materiales y métodos.}

El estudio se llevó a cabo en la comunidad de "LLacuas Huachac" que se encuentra ubicada en el Distrito de Manzanares, Provincia de Concepción, Región y Departamento de Junín, Perú. Fue realizado en tres fases, comenzando el 2006 y terminando el 2007.

El trabajo comenzó por la identificación de zonas homogéneas de producción de arveja, después fueron 
caracterizados los sistemas de manejo de suelos, en relación a los factores que limitaban o favorecían la sustentabilidad económica, ecológica y social del sistema.

Luego fueron seleccionados, un conjunto de indicadores para determinar el índice de sustentabilidad del sistema de manejo de suelos y puntos críticos. Finalmente se realizó las recomendaciones técnicas para efectuar diversos experimentos con el objetivo de mejorar los puntos críticos identificados e iniciar el manejo sustentable del suelo en la producción de arveja.

\section{Resultados y discusión.}

Zonas homogéneas de producción de arveja.

Dentro del territorio de la comunidad, cuyos suelos fueron clasificados por Garay (2001) en suelos A2cs y A3cst con 128.38 ha; A3cs con 360.82 ha; F3cst y F2c con 423.35 ha y como clase $X$ que incluye el avance urbano con 364.94 ha, se determinó dos zonas homogéneas de producción de arveja en base a la disponibilidad de riego (Figura 1):

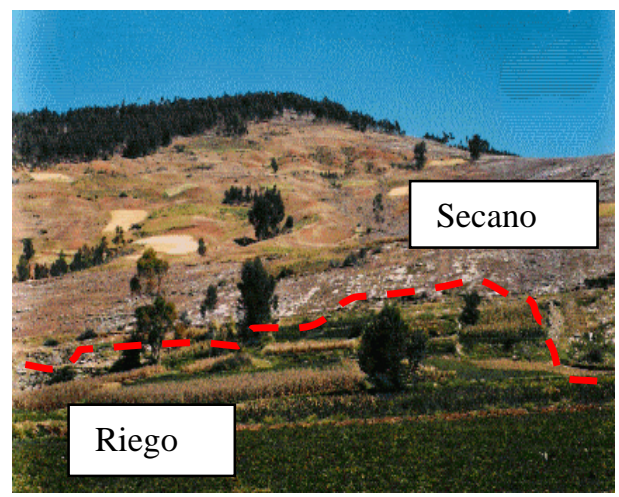

Figura 1. Las dos zonas homogéneas de producción.

a) Zona homogénea de producción con riego. Son lugares irrigables, que además de aprovechar la lluvia, son irrigados por medio de sistemas artificiales: canales, acequias, riego por aspersión y por goteo. Estas condiciones determinan un tipo de manejo de suelos dentro de una área aproximada de 128.38 ha (10.04\% de la superficie total), ubicados debajo de la línea del canal de irrigación que cruza el territorio de la comunidad de oeste a este. Esta zona homogénea de producción con riego, corresponde a la clase de suelos A2cs y A3cst. (Garay, 2001). Los cultivos predominantes en esta zona son alfalfa, cebolla, ajos, zanahoria, papa, maíz choclo, arveja para vaina en verde. Prevalece el sistema de cultivo con fertilización química y el de rotación con leguminosas.

b) Zona homogénea de producción en secano (sin riego). Son lugares donde las plantas crecen sin necesidad de riego artificial, reciben solo agua de la lluvia. Generando otro tipo de manejo de suelos. Es la zona ubicada en las laderas y zonas planas, por encima de la cota del canal de irrigación. Comprende aproximadamente 360.82 ha (28.24\%). Esta zona homogénea de producción sin riego corresponde a las clases de suelos A3cs (Garay, 2001). En donde se cultivan generalmente trigo, maíz amiláceo, cebada, quinua, arveja para grano seco. En esta zona prevalece el sistema de cultivos con descanso corto (1 año) y los conducidos con fertilización orgánica.

De los predios seleccionados.

Dos predios fueron seleccionados para realizar la evaluación de la sustentabilidad del manejo de suelos. El primero denominado "Huasipacha" de $400 \mathrm{~m}^{2}$, ubicado en la zona de producción en secano, y el segundo "Chaupimarca" de $600 \mathrm{~m}^{2}$, ubicada en la zona homogénea de producción con riego. Una característica común de ambos predios es que eran empleados para la siembra de arveja. El sistema de manejo de suelos de cada parcela seleccionada fue caracterizado en función a los indicadores de sustentabilidad del manejo de suelos y su relación con la producción de arveja.

Los indicadores de sustentabilidad del manejo de suelos.

La selección de los indicadores para la evaluación de la sustentabilidad del manejo de suelos en la producción de arveja, se realizó mediante la implementación de un proceso participativo (Arning, 2001).

Para ello se consideró: a) La caracterización, causas, efectos y factores limitantes del sistema de manejo de suelos en la producción de arveja; b) La facilidad de su uso práctico, medición e interpretación; y c) la sensibilidad y dependencia, a los cambios en las tecnologías de manejo de la calidad de los suelos (Brunett et al., 2005; Moreno et al., 2006). Además se tomaron en cuenta los requerimientos de sustentabilidad del manejo de suelos: a) diversidad de cultivos, b) suelo cubierto, c) labranza mínima, d) alta fijación biológica de nitrógeno atmosférico, e) alto contenido en materia orgánica, f) baja incidencia plagas y enfermedades y g) baja presencia de malezas (Sullivan, 2004).

De esta manera los indicadores de sustentabilidad del manejo de suelos en la producción de arveja identificados fueron: El carbono (C) y Nutrientes (N$\mathrm{P}_{2} \mathrm{O}_{5}-\mathrm{K}_{2} \mathrm{O}$ ) del suelo, densidad aparente (DA) de la capa arable, cobertura vegetal (CV), la presencia de malezas, el rendimiento (Rdto.), nodulación simbiótica de la arveja y la incidencia de plagas y enfermedades.

Análisis de los indicadores del manejo de suelos.

En la tabla 1 y figura 2, se observa que la reserva de carbono en el suelo con riego (83.53 $\mathrm{kg} \mathrm{ha}^{-1}$ ) fue menor que la de en secano (133.41 $\mathrm{kg} \mathrm{ha}^{-1}$ ). Ello por la mayor intensidad en la explotación del suelo bajo condiciones de riego. Así mismo el valor de la 
Tabla 1: Valores de los indicadores de sustentabilidad del manejo de suelos en la producción de arveja.

\begin{tabular}{lrrrrrrrr}
\hline $\begin{array}{l}\text { Indicador } \\
\text { Zona }\end{array}$ & $\begin{array}{l}\mathrm{C} \\
\mathrm{kg} \mathrm{ha}^{-1}\end{array}$ & $\begin{array}{l}\text { D.A. } \\
\mathrm{g} \mathrm{cm}^{-3}\end{array}$ & $\begin{array}{l}\mathrm{N} \mathrm{P}_{2} \mathrm{O}_{5} \mathrm{~K}_{2} \mathrm{O} \\
\mathrm{kg} \mathrm{ha}^{-1}\end{array}$ & $\begin{array}{l}\mathrm{C} \text {. V. } \\
\%\end{array}$ & $\begin{array}{l}\text { Malezas } \\
\mathrm{kg} \mathrm{m}^{-2}\end{array}$ & $\begin{array}{l}\text { Rdto } \\
\mathrm{kg} \mathrm{ha}^{-1}\end{array}$ & $\begin{array}{l}\text { Nodulación } \\
\mathrm{N}^{\mathrm{o}} \text { planta }^{-1}\end{array}$ & $\begin{array}{l}\text { Plagas-Enfer. } \\
\%\end{array}$ \\
\hline Con riego & 83.53 & 1.35 & $12-67-1322$ & 1 & 0.38 & 4055.06 & 9.5 & 5.56 \\
En secano & 133.41 & 1.30 & $18-144-603$ & 1 & 0.24 & 771.58 & 8.38 & 4.33 \\
\hline
\end{tabular}

densidad aparente de la capa arable de los suelos de la zona con riego $\left(1.35 \mathrm{~g} \mathrm{~cm}^{-3}\right)$ fue mayor, lo que indica que estos suelos presentan mayor grado de compactación, causados por el sobrepastoreo, labranza intensiva, transito de maquinaria y riegos pesados (gravedad). En ambas zonas los suelos tuvieron fertilidad media, con ausencia de cobertura vegetal, ello indica un mal manejo de la cobertura superficial del suelo y del mantenimiento de la fertilidad del suelo. La alta presencia de malezas en la zona con riego es otro de los indicadores del manejo inadecuado de los suelos. En la zona en secano el monocultivo, escaso abonamiento y rotación inadecuada tienen influencia significativa en los rendimientos. Así mismo se observó una escasa nodulación del cultivo de arveja y una alta incidencia de plagas y enfermedades.

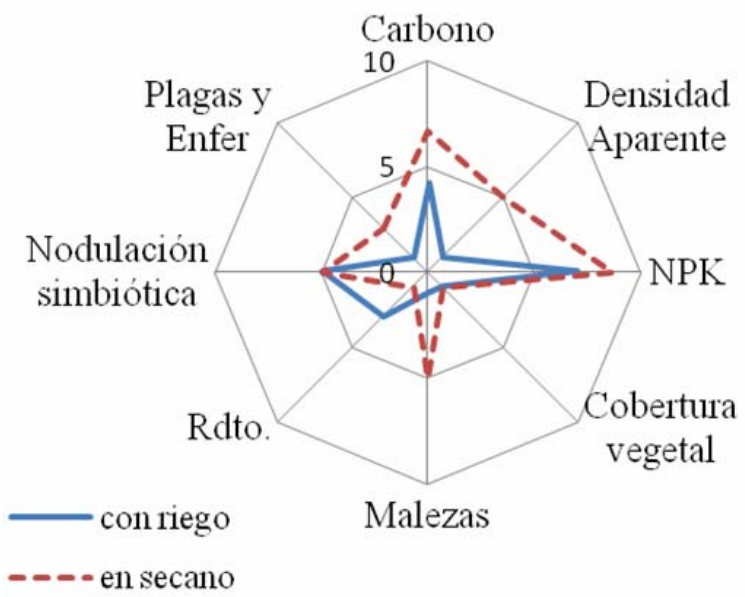

Figura 2. Los indicadores de sustentabilidad del manejo de suelos en los dos predios estudiados.

Bajo estas condiciones se concluye, que en ambas zonas los sistemas de manejo de suelos no son sustentables (Tabla 2), concordante con lo reportado por Díaz et al. (2005), quienes también encontraron que los sistemas de manejo convencionales de suelos no son sustentables.
Puntos críticos del manejo de suelos en la producción de arveja.

a) La remoción de los residuos de los cultivos, para combustible o forraje de ganados y el sobrepastoreo libre, exponen al suelo a la acción erosiva del viento y las precipitaciones pluviales, además interrumpe la restitución de los nutrientes hacia el suelo.

b) Escaso abonamiento, tanto con fertilizantes sintéticos y orgánicos.

c) El uso intensivo del suelo, sin descanso con deficiente rotación de cultivos altamente extractivos de nutrientes, disminuyen la fertilidad de los suelos, consecuentemente los suelos son de baja calidad.

d) La sobrepreparación del suelo mediante el empleo del arado convencional que contribuye fuertemente a la rápida pérdida de materia orgánica, compactación y formación de capas duras (claypans) y costras, característica típica del manejo de suelos en la zona con riego.

e) El riego inadecuado, riego por gravedad, y la falta de planificación y ejecución de programas de uso sostenible también influyen en la degradación del suelo.

Estrategias para el manejo sustentable del suelo.

De acuerdo con los resultados se puede sugerir la adopción de tecnologías de manejo alternativo de suelos, apropiadas a las condiciones reales de los diferentes Agroecosistemas. Entre estas tecnologías destacan los principios básicos del manejo de suelos sustentables, es decir el suelo debe ser perturbado lo menos posible, proteger permanentemente la superficie del suelo con cobertura vegetal, ya sea en forma viva o tipo mulch, incrementar el contenido de materia orgánica y nutrientes del suelo con mayor aporte de estiércol o compost de buena calidad combinados con fertilizantes químicos en cantidades adecuadas y establecer un programa de rotación, con secuencia adecuada y en función a los cultivos productores de materia orgánica y fijadores de nitrógeno atmosférico. Todas estas acciones deben complementarse entre si, para crear un suelo que tenga una mayor capacidad de absorción del agua de lluvia, mantener un suelo biológicamente rico, reducir la erosión y mantener un suministro de alimento continuo a las plantas, consecuentemente tener suelos de alta calidad, capaz de soportar un máximo de producción de cultivos de calidad y estable. 
Tabla 2. Calificación inicial de los indicadores y el índice de sustentabilidad del manejo de suelos en la producción de arveja.

\begin{tabular}{crrrrrrrrr}
$\begin{array}{c}* \text { Indicador } \\
\text { Zona }\end{array}$ & C & D A & NPK & C V & Malezas & $\begin{array}{c}\text { Rdto vaina } \\
\text { y grano }\end{array}$ & $\begin{array}{c}\text { Nodulación } \\
\text { Simbiótica }\end{array}$ & $\begin{array}{c}\text { Plagas } \\
\text {-Enfer. }\end{array}$ & $\begin{array}{c}\text { Indice } \\
\text { Sust } * *\end{array}$ \\
\hline Con riego & 4.19 & 1 & 7 & 1 & 1 & 3 & 5 & 1 & 2.90 \\
En secano & 6.67 & 5 & 8.67 & 1 & 5 & 1 & 5 & 3 & 4.42 \\
\hline
\end{tabular}

*Cada indicador fue estimado en forma separada, y se le asignó un valor de 1 a 10 , siendo 1 el valor menos deseable, 5 un valor moderado o medio y 10 el valor mas preferido.

**Para considerar que un sistema de manejo de suelos es sustentable el valor del índice de sustentabilidad (IS) debe ser mayor al valor del umbral (5) y además ninguno de los indicadores debe tener un valor menor a la del umbral de sustentabilidad (5).

\section{Conclusiones.}

De acuerdo con los resultados del presente trabajo, se puede concluir:

1. Existen dos zonas homogéneas de producción, una con riego y otra en secano. En la primera zona prevalecen los sistemas de cultivo con fertilización química y rotación con leguminosas. En la segunda zona prevalecen los sistemas de cultivos con descanso corto (1 año) y con fertilización orgánica.

2. El manejo de suelos en ambas zonas se caracteriza por la remoción de los residuos de los cultivos, reducida aplicación de fertilizantes orgánicos o inorgánicos y deficiente rotación de cultivos, a estos se suman la labranza intensiva y riego inadecuado en la zona conducida bajo riego.

3. Los indicadores de sustentabilidad del manejo de suelos en la producción de arveja identificados son: El carbono y nutrientes del suelo, densidad aparente de la capa arable, cobertura vegetal, la presencia de malezas, el rendimiento y nodulación simbiótica de la arveja, y la incidencia de plagas y enfermedades.

4. Los sistemas de manejos de suelos en ambas zonas de producción identificadas no son sustentables.

5. Los puntos críticos del manejo de suelos en ambas zonas son el manejo de cobertura vegetal, abonos, labranza y rotación de cultivos.

\section{Agradecimientos}

Al instituto Rural de Valle Grande de Cañete por su apoyo en los análisis de las muestras de suelos.

\section{Literatura citada.}

Altieri M.A. \& Nicholls C.I. 2007. Conversión agroecológica de sistemas convencionales de producción: teoría, estrategias y evaluación. AEET. Ecosistemas. España. 16(1): 3-12.

Altieri M.A. \& Nicholls C.I. 2005. A rapid, farmer-friendly agroecological method to estimate soil quality and crop health in vineyard systems. Agroecology and the search for a truly sustainable agriculture. PNUMA. : 277-290

Apollin F. \& Eberhart C. 1999. Análisis y diagnóstico de los sistemas de producción en el medio rural. CICDA RURALTER. CAMAREN. Quito - Ecuador.

Arning I. 2001. Guía metodológica para investigadores agrícolas: Introducción práctica a la investigación participativa e investigación científica. RAAA.LimaPerú.

Brunett P.L., González E.C. \& García H.L.A. 2005. Evaluación de la sustentabilidad de dos agroecosistemas campesinos de producción de maíz y leche, utilizando indicadores. Livestock research for rural development. 17(7), article 78.

CARITAS. 2004. Manual del cultivo de arveja. Caritas Huancayo, INIA, UNCP, Fondo Italo Peruano. Huancayo - Perú.

Cufre G., Rodriguez C. \& Pagliaricci H. 2002. Sustentabilidad. FAV UNRC. www.produccionanimal.com.ar.

Díaz B., Cairo P., Rodríguez O., Abreu I., Torres P., Jiménez R., Dávila A. \& Colas A. 2005. Evaluación de la sostenibilidad del manejo del suelo pardo con carbonato (inceptisol) a través de indicadores de calidad del mismo. Centro agrícola. 32 (2): 73-78.

Fitz P.E.A. 1993. Suelos: su formación, clasificación, distribución. CECSA. 5ta reimpresión. México.

Garay G.J.O. 2001. Evaluación de los sistemas de producción agropecuaria en la comunidad campesina de Llacuas Huachac. Tesis Ing. Agr. Huancayo-Perú. UNCP. Facultad De Agronomía.

MINAG (Ministerio de Agricultura, PE). 2007. Datos estadísticos de siembra. Disponible desde internet en: http//web. Minag.gob.pe.

Moreno H.A., Pedraza G.X. \& Solarte A.J. 2006. Construcción y uso de indicadores de sustentabilidad para la planeación participativa de predios. www.ecoportal.net.

Nario M.A., Pino N.I., Parada C.A.M., Rouanet M.J.L., Barrientos L. \& Montenegro A. 2001. Efecto de rotación de cultivos en el balance de nitrógeno (15N), en labranza conservacionista. R.C. Suelo Nutr. Veg. [online]. dic. 2001, vol.1, no.2 [citado 29 Marzo 2009], p. 42-48. Disponible en la World Wide Web: $<$ http://mingaonline.uach.cl/scielo.php? script=sci_arttext\&pid=S071827912001000200005\&lng=es\&nrm=iso>. ISSN 07182791.

Nuñez M.A. 2005. Bases científicas de la agricultura tropical sustentable. Motion Magazine. Barinas, 
Venezuela.

Santillana V.N. 2006. Producción de biofertilizantes utilizando Pseudomonas sp. Ecología Aplicada. 5(1,2): 87-91.

Sullivan P. 2004. Sustainable soil management: Soil systems guide. ATTRA. IP027. Slot 133. Versión 062104. USDA (Department of Agriculture, US). 1994. Manual de conservación de suelos. Editorial LIMUSA, S.A.8va. Reimpresión. México.

${ }^{1}$ Universidad Nacional de Huancavelica. Facultad de Ciencias Agrarias. agustinperales60@hotmail.com.

${ }^{2}$ Universidad Nacional Agraria La Molina. Facultad de Agronomía. Departamento Académico de suelos. ololi@lamolina.edu.pe.

${ }^{3}$ Universidad Nacional Agraria La Molina. Facultad de Agronomía. Departamento Académico de suelos. jalegre@lamolina.edu.pe.

${ }^{4}$ Universidad Nacional Agraria La Molina. Facultad de Agronomía. Departamento Académico de Fitotecnia. camafe@lamolina.edu.pe. 